\title{
A CLASS OF DISCREPANCY PRINCIPLES FOR THE SIMPLIFIED REGULARIZATION OF ILL-POSED PROBLEMS
}

\author{
SANTHOSH GEORGE and M. THAMBAN NAIR ${ }^{1}$
}

(Received 30 April 1993; revised 11 May 1993)

\begin{abstract}
A class of discrepancy principles for the choice of parameters for the simplified regularization of ill-posed problems is proposed. This procedure does not require knowledge of the unknown solution, and if the smoothness of the unknown solution is known then the convergence rate obtained is optimal. The results of this paper include the Arcangeli's method considered by Groetsch and Guacaneme (1987) for which the convergence rate was not known and also of a result of Guacaneme (1988) for which there is a gap in the proof.
\end{abstract}

\section{Introduction}

Many inverse problems of mathematical physics and problems of remote sensing lead to the solution of a Fredholm integral equation of the first kind,

$$
\int_{a}^{b} k(s, t) x(t) d t=y(s), \quad a \leq s \leq b,
$$

where the kernel $k(.,$.$) is a nondegenerate square integrable function and y($.$) is a$ known 'data' function. This problem is, in general, ill-posed in the sense that even if a unique solution exists, the solution need not depend continuously on the data $y$. Using operator notation the above equation takes the form

$$
T x=y
$$

with operator $T: L^{2}[a, b] \rightarrow L^{2}[a, b]$ defined by

$$
(T x)(s)=\int_{a}^{b} k(s, t) x(t) d t, \quad a \leq s \leq b .
$$

\footnotetext{
'Department of Mathematics, Goa University, Goa - 403 203, India

(C) Australian Mathematical Society, 1994, Serial-fee code 0334-2700/94
} 
The operator $T$ is compact, and since the kernel $k(.,$.$) is nondegenerate its range$ $R(T)$ is not closed in $L^{2}[a, b]$ which results in the ill-posedness of (1) (see [4]).

A well known method for the regularization of (1) is the Tikhonov regularization where the solution $x_{\alpha}$ of

$$
\left(T^{*} T+\alpha I\right) x_{\alpha}=T^{*} y, \quad \alpha>0
$$

is taken as an approximation of $x$. This procedure is considered extensively in the literature. (See Groetsch [4] and the references therein.) We note that $T^{*} T$ is a positive semi definite operator so that (2) is well posed in the usual sense, that is, for each $\alpha>0$, (2) is uniquely solvable and the solution depends continuously on the data $y$.

If the kernel $k(.,$.$) is symmetric, that is, k(s, t)=k(t, s)$ then the operator $T$ itself is a positive semi-definite operator and in that case a simplified regularization procedure can be used instead of the Tikhonov regularization. This procedure, introduced by Schock [11], has computational advantages over the Tikhonov regularization. We shall consider this in the general frame work of operators on a Hilbert space.

Let $A$ be a positive semi definite operator on a Hilbert space $H$ with non closed range $R(A)$ and $g \in R(A)$. Consider the operator equation

$$
A w=g .
$$

The non closedness of the range $R(A)$ leads to the ill posedness of (3). (See Groetsch [4].) We note that taking $A=T T^{*}, g=y$ and $x=T^{*} w$ the equation (1) takes the form (3). Also the normal form of (1), namely $T^{*} T x=T^{*} y$, is of the form (3) with $A=T^{*} T$ and $g=T^{*} y$. We consider the simplified regularization of (3), where the solution $w_{\alpha}$ of the well-posed problem

$$
(A+\alpha I) w_{\alpha}=g, \quad \alpha>0
$$

is taken as an approximation. It is shown in [11] that $w_{\alpha} \rightarrow w$ as $\alpha \rightarrow 0$ and $\left(w_{\alpha}\right)$ has better convergence properties than the approximation obtained by the Tikhonov regularization of (1). Here $w$ is the unique element in the orthogonal complement of the null space of $A$ such that $A w=g$. It is also known (Schock [9]) that if $w \in R\left(A^{v}\right)$, $0<v \leq 1$, then

$$
\left\|w-w_{\alpha}\right\|=O\left(\alpha^{\nu}\right) .
$$

In practical situations one may not have the data $g$ exactly, instead one may have $g^{\delta}$ such that $g^{\delta} \rightarrow g$ as $\delta \rightarrow 0$. In that case we replace the right-hand side of (4) by $g^{\delta}$ and consider the corresponding solution $w_{\alpha}^{\delta}$, that is, $w_{\alpha}^{\delta}$ is the unique solution determined by

$$
(A+\alpha I) w_{\alpha}^{\delta}=g^{\delta} .
$$


The point now is to choose the regularization parameter $\alpha=\alpha(\delta)$ depending on the error level $\delta$ such that $\alpha(\delta) \rightarrow 0$ and $w_{\alpha(\delta)}^{\delta} \rightarrow w$ as $\delta \rightarrow 0$. We assume that $\left\|g-g^{\delta}\right\| \leq \delta$. It is known (Schock [9]) that if $w \in R\left(A^{\nu}\right), 0<v \leq 1$, and $\alpha(\delta)=c \delta^{1 /(\nu+1)}$, then

$$
\left\|w-w_{\alpha}^{\delta}\right\|=O\left(\delta^{v /(v+1)}\right),
$$

and this rate is optimal. In a posteriori choice strategies, the parameter $\alpha$ is determined during the course of computation of $w_{\alpha}^{\delta}$. Such methods are considered by many authors for the Tikhonov regularization and its general forms. (See, for example, Engl [1], Engl and Neubauer [3], Engl and Gfrerer [2], Schock [10], Nair [8], and the references therein). For the simplified regularization of (3), Groetsch and Guacaneme [5] considered an analogue of Arcangeli's method, namely,

$$
\left\|A w_{\alpha}^{\delta}-g^{\delta}\right\|=\delta / \sqrt{\alpha},
$$

and proved that if $\alpha=\alpha(\delta)$ is chosen according to (7) and $A$ is, in addition, a compact operator, then $w_{\alpha}^{\delta} \rightarrow w$ as $\delta \rightarrow 0$. But no attempt has been made to obtain estimates for the error $\left\|w-w_{\alpha}^{\delta}\right\|$. It is the purpose of this paper to prove the convergence and also to obtain error estimate under a general class of discrepancy principles,

$$
\left\|A w_{\alpha}^{\delta}-g^{\delta}\right\|=\frac{\delta^{p}}{\alpha^{q}}, \quad p>0, q>0,
$$

which is valid for $0<p<q+1$. If the smoothness of the solution $w$ is known, namely, $w \in R\left(\alpha^{v}\right), 0<v \leq 1$, then by taking $p=(q+1) /(\nu+1)$ our result provides the optima1 rate $O\left(\delta^{\nu /(v+1)}\right)$. As a particular case, the discrepancy principle (7) gives the rate $O\left(\delta^{1 / 3}\right)$, and the best rate $O\left(\delta^{1 / 2}\right)$ is obtained when $v=1$ by taking $p=(q+1) / 2$.

The result for the case $v=1$ has also been considered by Guacaneme [6] which came to the notice of the authors after they communicated the first draft of the paper. In fact, the proof of the main result of Guacaneme ([6, Theorem 2.3]) is not complete as he uses the estimate $O\left(\delta^{1-p /(q+1)}\right)$ for $\delta / \alpha$ which is not immediate from the estimate $\alpha=O\left(\delta^{p /(q+1)}\right)([6$, Lemma 2.1$])$.

\section{The Main Results}

For the determination of the regularization parameter $\alpha=\alpha(\delta)$ for the simplified regularization of (3) with inexact data $g^{\delta}$ such that $\left\|g-g^{\delta}\right\| \leq \delta$, we consider the discrepancy principle

$$
\left\|A w_{\alpha}^{\delta}-g^{\delta}\right\|=\frac{\delta^{p}}{\alpha^{q}}, \quad p>0, q>0,
$$

where $w_{\alpha}^{\delta}$ is the solution of (6). We assume that $0 \neq g \in R(A)$. For given $q>0$, let

$$
\phi(\alpha)=\alpha^{2 q}\left\|A w_{\alpha}^{\delta}-g^{\delta}\right\|^{2}, \quad \alpha>0 .
$$


LEMMA 1. The function $\phi(\alpha)$ is continuous and strictly increasing for $\alpha>0$, and satisfies $\lim _{\alpha \rightarrow 0} \phi(\alpha)=0$ and $\lim _{\alpha \rightarrow \infty} \phi(\alpha)=\infty$.

PROOF. We observe that

$$
\begin{aligned}
\phi(\alpha) & =\alpha^{2 q}\left\langle A w_{\alpha}^{\delta}-g^{\delta}, A w_{\alpha}^{\delta}-g^{\delta}\right\rangle \\
& =\alpha^{2 q+2}\left\|w_{\alpha}^{\delta}\right\|^{2} \\
& =\alpha^{2 q+2}\left\langle(A+\alpha I)^{-2} g^{\delta}, g^{\delta}\right\rangle
\end{aligned}
$$

It is seen that

$$
\frac{\alpha^{2 q+2}\left\|g^{\delta}\right\|^{2}}{\|(A+\alpha I)\|^{2}} \leq \phi(\alpha) \leq \alpha^{2 q}\left\|g^{\delta}\right\|^{2}
$$

and

$$
\begin{aligned}
\phi^{\prime}(\alpha) & =\alpha^{2 q+1}\left\{2 q\left\langle(A+\alpha I)^{-2} g^{\delta}, g^{\delta}\right\rangle+2\left\langle(A+\alpha I)^{-3} A g^{\delta}, g^{\delta}\right\rangle\right\} \\
& >0
\end{aligned}
$$

for $\alpha>0$. Thus $\phi(\alpha)$ is continuous for $\alpha>0, \phi(\alpha) \rightarrow 0$ as $\alpha \rightarrow 0, \phi(\alpha) \rightarrow \infty$ as $\alpha \rightarrow \infty$ and $\phi(\alpha)$ is strictly increasing for $\alpha>0$.

LEMMA 2. For each $\delta>0$, there exists a unique $\alpha=\alpha(\delta)$ satisfying (8). Further $\alpha(\delta) \rightarrow 0$ as $\delta \rightarrow 0$.

Proof. By Lemma 1 and the intermediate value theorem, it follows that there exists a unique $\alpha=\alpha(\delta)$ satisfying (8). The result $\alpha(\delta) \rightarrow 0$ as $\delta \rightarrow 0$ follows using the arguments as in Schock ([10, Lemma 1]).

THEOREM 3. If $\alpha=\alpha(\delta)$ is chosen according to (8), then $\alpha(\delta)=O\left(\delta^{p /(q+1)}\right)$. If, in addition, $p<q+1$, then $\delta / \alpha(\delta)=O\left(\delta^{m}\right), m=(q+1-p) /(q+1)$, and $w_{\alpha}^{\delta} \rightarrow w$ as $\delta \rightarrow 0$.

PROOF. First we note that

$$
\begin{aligned}
\left\|g^{\delta}\right\|-\frac{\delta^{p}}{\alpha^{q}} & =\left\|g^{\delta}\right\|-\left\|A w_{\alpha}^{\delta}-g^{\delta}\right\| \\
& \leq\left\|A w_{\alpha}^{\delta}\right\| \\
& =\frac{\left\|A\left(A w_{\alpha}^{\delta}-g^{\delta}\right)\right\|}{\alpha} \\
& \leq\|A\| \frac{\delta^{p}}{\alpha^{q+1}} .
\end{aligned}
$$


Therefore,

$$
\alpha^{q+1} \leq \frac{\delta^{p}(\|A\|+\alpha)}{\left\|g^{\delta}\right\|},
$$

so that

$$
\alpha=O\left(\delta^{p /(q+1)}\right)
$$

We note that

$$
\frac{\delta^{p}}{\alpha^{q}}=\left\|A w_{\alpha}^{\delta}-g^{\delta}\right\|=\left\|\alpha w_{\alpha}^{\delta}\right\| \leq \alpha\left(\left\|w_{\alpha}^{\delta}-w_{\alpha}\right\|+\left\|w_{\alpha}\right\|\right) .
$$

But $w_{\alpha}-w_{\alpha}^{\delta}=(A+\alpha I)^{-1}\left(g-g^{\delta}\right)$ so that

$$
\left\|w_{\alpha}-w_{\alpha}^{\delta}\right\| \leq \frac{\delta}{\alpha}
$$

Also $\left\|w_{\alpha}\right\|=\left\|(A+\alpha I)^{-1} A w\right\| \leq\|w\|$. Therefore we obtain

$$
\frac{\delta^{p}}{\alpha^{q}} \leq \alpha\left(\frac{\delta}{\alpha}+\|w\|\right)=\delta+\alpha\|w\| .
$$

Now using the estimate $\alpha=O\left(\delta^{p /(q+1)}\right)$, we get

$$
\begin{aligned}
\frac{\delta}{\alpha} & =\delta^{1-p / q}\left(\delta^{p} / \alpha^{q}\right)^{1 / q} \\
& =\delta^{1-p / q}(\delta+\alpha\|w\|)^{1 / q} \\
& \leq\left(\delta^{q-p+1}+c \delta^{q-p+p /(q+1)}\right)^{1 / q}
\end{aligned}
$$

and since $p<q+1$

$$
\frac{\delta}{\alpha}=O\left(\delta^{m}\right)
$$

where $m=(q+1-p) /(q+1)$. To prove the convergence we first note that $\left\|w-w_{\alpha}^{\delta}\right\| \leq\left\|w-w_{\alpha}\right\|+\left\|w_{\alpha}-w_{\alpha}^{\delta}\right\|$. Let $R_{\alpha}=\alpha(A+\alpha I)^{-1}$. Then $w-w_{\alpha}=R_{\alpha} w$, so that using (10) and (11) it is enough to prove that $R_{\alpha(\delta)} w \rightarrow 0$ as $\delta \rightarrow 0$. But $\left\|R_{\alpha}\right\| \leq 1$ for every $\alpha>0$ and for any $u \in R(A),\left\|R_{\alpha} u\right\|=\left\|R_{\alpha} A v\right\| \leq \alpha\|v\|$ for $u=A v$, so that $R_{\alpha} u \rightarrow 0$ as $\alpha \rightarrow 0$ for every $u$ in $R(A)$. Therefore by using the fact that $R(A)$ is a dense subspace of the orthogonal complement of the null space of $A$ and $\left\|R_{\alpha}\right\| \leq 1$, it follows that $R_{\alpha(\delta)} w \rightarrow 0$ as $\delta \rightarrow 0$. This completes the proof of the theorem.

THEOREM 4. Let $w \in R\left(A^{v}\right), \theta<\nu \leq 1, q>0, p<q+1$ and $\alpha=\alpha(\delta)$ be chosen according to (8). Then

$$
\left\|w-w_{\alpha}^{\delta}\right\|=O\left(\delta^{s}\right)
$$


where $s=\min \{p v /(q+1), 1-p /(q+1)\}$. For a fixed $v$ the best rate is obtained when $p=(q+1) /(v+1)$ which gives $\alpha=O\left(\delta^{1 /(v+1)}\right)$ and

$$
\left\|w-w_{\alpha}^{\delta}\right\|=O\left(\delta^{v /(v+1)}\right)
$$

PROOF. From (5) and (10) we have

$$
\left\|w-w_{\alpha}^{\delta}\right\| \leq\left\|w-w_{\alpha}\right\|+\left\|w_{\alpha}-w_{\alpha}^{\delta}\right\|=O\left(\alpha^{v}\right)+O(\delta / \alpha),
$$

so that the result in (i) follows from Theorem 3. If $p=(q+l) /(\nu+1)$ then $p \nu /(q+1)=(q+1-p) /(q+1)$ so that $O\left(\alpha^{\nu}\right)=O(\delta / \alpha)=O\left(\delta^{\nu /(v+1)}\right.$, proving (ii).

COROLlaRY 5. If $w \in R\left(A^{\nu}\right), 0<\nu \leq 1$ and $\alpha=\alpha(\delta)$ is chosen according to (7), then

$$
\left\|w-w_{\alpha}^{\delta}\right\|=O\left(\delta^{k}\right)
$$

where $k=\min \{2 \nu / 3,1 / 3\}$.

REMARK. From Theorem 4 it follows that the best rate $O\left(\delta^{1 / 2}\right)$ is achieved for $w \in$ $R(A)$ by taking $p=(q+1) / 2$, in particular for $p=1=q$.

In the case of the general ill-posed problem (1), if $A=T T^{*}, g=y, g^{\delta}=y^{\delta}$ and $x_{\alpha}^{\delta}=T^{*} w_{\alpha}^{\delta}$, then $x_{\alpha}^{\delta}$ is the Tikhonov regularized solution of (3) with $y^{\delta}$ in place of $y$, and the discrepancy principle (8) is the same as the one considered by Schock [10] and subsequently by Guacaneme [7] and Nair [8], namely $\left\|T x_{\alpha}^{\delta}-y^{\delta}\right\|=\delta^{p} / \alpha^{q}$. But the estimate in Theorem 4 does not help directly to deduce the results in [10], [7] and [8].

If we use a different definition of the noise level, namely, $\left\|y-y^{\delta}\right\| \leq \delta / c$ with $\left\|T^{*}\right\| \leq c$, then the discrepancy principle $\left\|T^{*} T x_{\alpha}^{\delta}-T^{*} y^{\delta}\right\|=\delta^{p} / \alpha^{q}$ considered by Engl [1] and Engl and Neubauer [3] is of the form (8) with $A=T^{*} T, g=T^{*} y$, $g^{\delta}=T^{*} y^{\delta}$ and $w_{\alpha}^{\delta}=x_{\alpha}^{\delta}$. The estimate $\alpha=O\left(\delta^{p /(q+1)}\right)$ of Theorem 3 can be used to obtain the optimal estimate of [1] and [3] as follows :

We observe that

$$
w_{\alpha}-w_{\alpha}^{\delta}=(A+\alpha I)^{-1}\left(g-g^{\delta}\right)=\left(T^{*} T+\alpha\right)^{-1} T^{*}\left(y-y^{\delta}\right)
$$

so that $\left\|w_{\alpha}-w_{\alpha}^{\delta}\right\|=O(\delta / \sqrt{\alpha})$ and hence from (9),

$$
\begin{aligned}
\frac{\delta^{p}}{\alpha^{q}} & =O(\delta \sqrt{\alpha}+\alpha) \\
& =O\left(\delta^{p /(q+1)}\right)
\end{aligned}
$$

for $p<2(q+1)$, and hence

$$
\frac{\delta}{\sqrt{\alpha}}=\delta^{1-p / 2 q}\left(\frac{\delta^{p}}{\alpha^{q}}\right)^{\frac{1}{2 q}}=O\left(\delta^{1-p / 2(q+1)}\right) .
$$


Therefore if $p<2(q+1)$ and $0<v \leq 1$, then we have

$$
\left\|w-w_{\alpha}^{\delta}\right\|=O\left(\delta^{h}\right)
$$

where $h=\min \{p v /(q+1), 1-p / 2(q+1)\}$, so that the optimal estimate $O\left(\delta^{2 v /(2 v+1)}\right)$ is achieved for $p=2(q+1) /(2 v+1)$.

In general the simplified regularization is recommended when the operator $T$ under consideration is positive semi-definite, because in this case the methods in [1], [10] etc, involve more computation as for such operators we have $T T^{*}=T^{2}=T^{*} T$.

\section{Acknowledgements}

The authors thank the referees for their valuable comments on the first version of this paper and also for suggesting to include in the references the papers of Engl, Engl and Neubauer and Guacaneme. The work of Santhosh George is supported by the Junior Research Fellowship of the C.S.I.R., India.

\section{References}

[1] H. W. Engl, "Discrepancy principles for Tikhonov regularization of ill-posed problems leading to optimal convergence rates", J. Optim. Theory Appl. 52 (1987) 209-215.

[2] H. W. Engl and H. Gfrerer, "A posteriori parameter choice for the general regularization methods for solving linear ill-posed problems", Appl. Numer. Math. 4 (1988) 395-417.

[3] H. W. Engl and A. Neubauer, "Optimal discrepancy principle for the Tikhonov regularization of integral equations of the first kind", in Constructive methods for the Practical Treatment of Integral Equations (eds. G. Hammerlin and K. H. Hoffman), ISNM 73, (Birkhauser Verlag, Basel, 1985) $120-141$.

[4] C. W. Groetsch, The theory of Tikhonov regularization method for Fredholm integral equations of the first kind (Pitman, Boston, 1984).

[5] C. W. Groetsch and J. Guacaneme, "Arcangeli's method for Fredholm equations of the first kind", Proc. Amer. Math. Soc. 99 (1987) 256-260.

[6] J. E. Guacaneme, "On simplified regularization", J. Optim. Theory Appl. 58 (1988) 133-138.

[7] J. E. Guacaneme, "An optimal parameter choice for regularized ill-posed problems", Integral Equations Operator Theory 11 (1988) 610-613.

[8] M. Thamban Nair, "A generalization of Arcangeli's method for ill-posed problems leading to optimal rates", Integral Equations Operator Theory 15 (1992) 1042-1046.

[9] E. Schock, "On the asymptotic order of accuracy of Tikhonov regularization", J. Optim. Theory Appl. 44 (1984) 95-104.

[10] E. Schock, "Parameter choice by discrepancy principles for the approximate solution of ill-posed problems", Integral Equations Operator Theory 7 (1984) 895-898.

[11] E. Schock, "Ritz regularization versus least square regularization: Solution methods for integral equations of the first kind", Zeitschrift für Analysis und ihre Anwedungen 2 (1985) . 